\section{An ingenious two-in-one solution}

For effective obturation of the root canal, COLTENE is pleased to introduce the ROEKO GuttaFlow bioseal, which combines free-flow gutta-percha with sealer to provide an ingenious two-in-one solution.

As well as excellent flow properties and radiopacity, a tight seal and optimal protection against re-infection, GuttaFlow bioseal actively supports regeneration in the root canal by providing the tooth with natural repair constituents and activating biochemical processes.

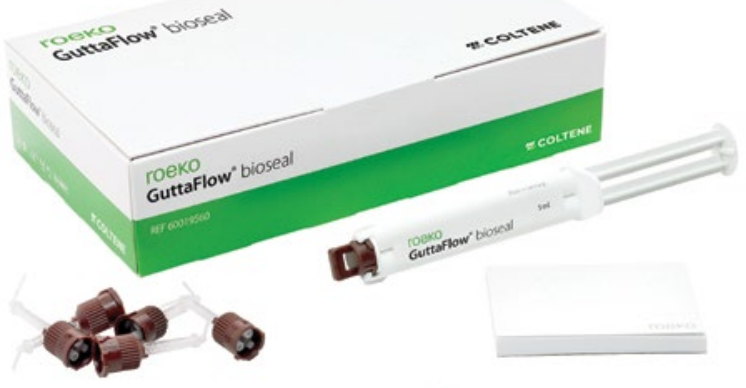

Apart from MTA and bioglass, GuttaFlow bioseal is the only material known to exhibit these regenerative and supportive properties - the difference is that GuttaFlow doesn't have long curing times or complicated handling.

For a two-in-one solution that does it all, contact COLTENE.

To find out more visit www.coltene.com, email info.uk@ coltene.com or call 01444235486.

\section{A membership with benefits for all}

The Association of Dental Implantology (ADI) offers its membership a myriad of benefits to enjoy throughout their careers. For ADI Clinician and First Five Years members, these include:

- Free premium membership of Dentinal Tubules

- Members' National Forum - free to attend!

- Free ADI Study Clubs

- Discounts on ADI events such as the ADI Team Congress

- Free access to prestigious research journals

- Members-only Facebook Group.

With all this and more, ADI membership really does offer something to suit everyone. Find out how joining a community of forward-thinking professionals could benefit you and your career development today.

For more information or to join the ADI, please visit www.adi. org.uk.

\section{Hands-on dynamic navigation training}

Implant dentists who want to take precision and safety to a new level can learn how with Master Clinical Trainer, Dr David Burgess. The Dynamic Navigation Society's UK training programme is entering its fifth year and includes regular one-day introductory courses in freehand surgery using real-time computer guidance. Delegates are given an overview of the complete process from patient diagnosis to navigated implant treatment. Bespoke two-day masterclasses offer training specifically tailored to the attendee's requirements.

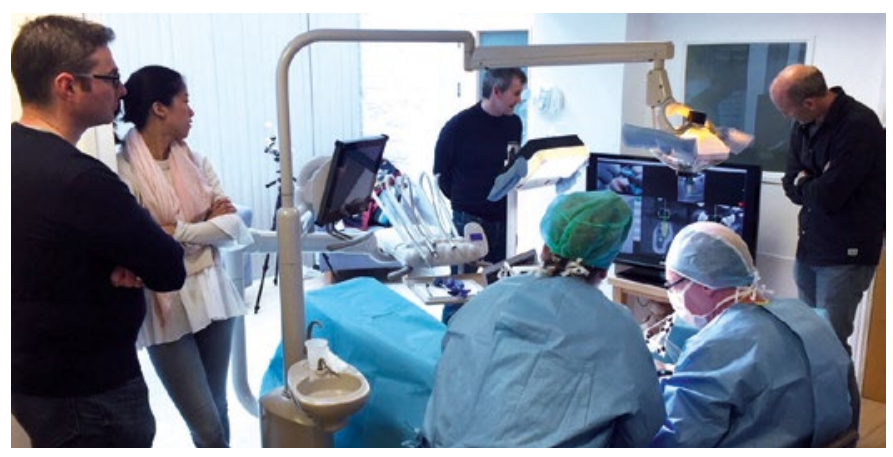

Participants have the opportunity to prepare dental implant sites on models and phantom heads with dynamic navigation technology. Dr Arshad Ali, Clinical Director of the Scottish Centre of Excellence for Dentistry, attended the one-day course and described it as an 'excellent course with many practical tips, given by an exceptional clinician who I can see is supported by an incredible team!'

Delegates observe case planning and live surgery using the Navident system, learning about the digital dentistry workflow and clinical protocol.

The two-day masterclass is aimed at dentists who already use dynamic navigation and want to accelerate integration into their everyday practice. The course provides a comprehensive insight into the benefits of freehand surgery with real-time computer guidance.

Details of the latest courses can be obtained by calling 0845602 4944, emailing info@dynamicnavigationsociety.org.uk or at http://bit. ly/DNSUKcourses. Courses are limited to five delegates for maximum benefit. Early bird discounts apply.

\section{So much more than it says on the tin}

The British Dental Conference and Dentistry Show 2020 is the ideal platform for all members of the dental team to develop their knowledge, gain CPD and source the latest products.

The next event will once again present an array of world-class speakers, each offering a wealth of information and practical advice. Sessions will cover clinical and business topics, with enhanced CPD available for all.

Further still, the trade floor will host more than 450 manufacturers and suppliers, offering demonstrations, guidance and on-stand learning opportunities.

The British Dental Conference and Dentistry Show 2020 will take place on 15 and 16 May -Birmingham NEC, co-located with DTS.

For all the latest information, visit www.thedentistryshow.co.uk, call 02073485270 or email dentistry@closerstillmedia.com. 